\title{
Histotripsy of Rabbit Renal Tissue in Vivo: Temporal Histologic Trends
}

\author{
TIMOTHY L. HALL, Ph.D., ${ }^{1}$ KATHLEEN KIERAN, M.D., ${ }^{2}$ KIMBERLY IVES, D.V.M., ${ }^{1}$ \\ J. BRIAN FOWLKES, Ph.D., ${ }^{1}$ CHARLES A. CAIN, Ph.D., ${ }^{1}$ and WILLIAM W. ROBERTS, M.D. ${ }^{2}$
}

\begin{abstract}
Background and Purpose: Histotripsy is defined as noninvasive, nonthermal, mechanical (cavitational) tissue ablation. We previously demonstrated the predictable acute tissue effects of histotripsy in rabbit kidney and other tissues. We sought to characterize the appearance and natural history of renal tissue after histotripsy.

Materials and Methods: Following Institutional Animal Care Committee approval, the left kidneys of 29 rabbits were treated with $60,000750-\mathrm{kHz}, 15$-cycle bursts of ultrasound energy from an 18-element phasedarray transducer at a 1-kHz pulse-repetition frequency. The treated kidneys were harvested at $0,1,2,7,21$, or 60 days; fixed in Formalin; then prepared for microscopic analysis with hematoxylin and eosin and trichrome stains.

Results: For kidneys harvested acutely (day 0 ), a contiguous area of finely disrupted tissue was observed containing no recognizable cells or cellular components. Along the boundary of architectural disruption, a border several tubules wide contained cells that were not visibly disrupted but appeared damaged (pyknotic nuclei). At subsequent time intervals, an inflammatory response developed in association with a steadily decreasing area of cellular and architectural disruption. By day 60, only a small fibrous scar persisted adjacent to a wedge of tubular dilation and fibrosis underlying a surface-contour defect.

Conclusions: Histotripsy produces mechanical fractionation of cellular and architectural structures. The resultant acellular material appears to be readily reabsorbed within 60 days in the rabbit. This may prove to be a significant advantage for imaging assessment of residual tumor after ablation of renal malignancy.
\end{abstract}

\section{INTRODUCTION}

A N ESTIMATED 51,190 PATIENTS in the United States will be found to have renal cancer in $2007 .{ }^{1}$ As a result of the widespread use of cross-sectional imaging, these renal masses frequently are identified when smaller and at an earlier stage of disease than in the past. Many of these tumors are amenable to newer minimally invasive thermal ablative therapies such as cryoablation and radiofrequency ablation (RFA), which are less-morbid alternatives to traditional surgical therapy (radical or partial nephrectomy). With these modalities, energy is delivered percutaneously to either freeze the tissue and fracture the cell membranes or to heat the tissue to produce coagulative necrosis.

Noninvasive thermal ablation with focused ultrasound, commonly used in Europe and Asia for transrectal prostate ablation, ${ }^{2-4}$ also has been used experimentally for transcutaneous treatment of renal masses. 5,6 Typical treatment methods use an intense 1- to 20-second burst of ultrasound energy to induce frictional heating of tissue within the focal volume. Once a sufficient thermal dose is achieved, coagulative necrosis and immediate cell death occur. ${ }^{2-7}$ As the focal volume typically is small (e.g., a cylinder $10 \mathrm{~mm}$ in length and $3 \mathrm{~mm}$ in diameter), the ultrasound focus is moved through the target tissue to build up a larger treatment volume.

Focused ultrasound also can be delivered as short $(20-\mu \mathrm{sec})$ pulses with greater intensity and short $(<4 \%)$ duty cycles to generate a nonthermal mechanical (cavitational) bioeffect that physically disrupts tissue. ${ }^{8-11} \mathrm{We}$ have named this process "histotripsy" and have demonstrated the feasibility of this technique for transcutaneous ablation of renal tissue in an acute rabbit model. ${ }^{11}$ An incremental increase in the fraction of tissue disruption was observed as the number of pulses was increased from 10 to 10,000. Application of 10,000 pulses produced com-

Departments of ${ }^{1}$ Biomedical Engineering and ${ }^{2}$ Urology, University of Michigan, Ann Arbor, Michigan. 


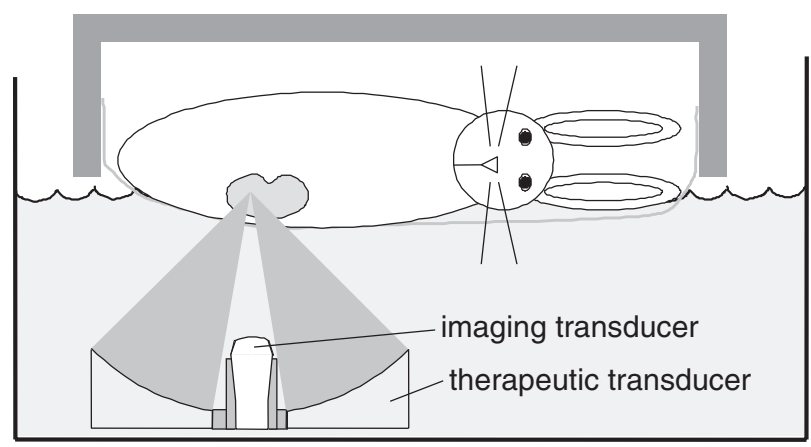

FIG. 1. Sketch of experimental set-up. Rabbits were placed on thin plastic membrane suspended over tank of degassed water containing therapeutic transducer.

plete tissue fractionation within the focal zone, leaving a uniform acellular liquid surrounded by a narrow margin of partial disruption. ${ }^{11}$

In this study, we sought to characterize the appearance and natural history of renal tissue after treatment with histotripsy. We hypothesized that the fractionated material would be reabsorbed rapidly with minimal scar formation. This study was approved by the Institutional Animal Care Committee of our institution.

\section{MATERIALS AND METHODS}

Rabbits were prepared for ablation by anesthetizing them with intramuscular injections of ketamine $35 \mathrm{mg} / \mathrm{kg}$ and $\mathrm{xy}-$ lazine $5 \mathrm{mg} / \mathrm{kg}$. Depilatory creams had been found to cause severe skin irritation, so a two-step shaving process for hair removal was used instead. Ophthalmic ointment (VEDCO, St. Joseph, MO) was applied. The abdomen was shaved with electric clippers to remove the majority of the hair and then a second time with an electric facial shaver to remove the remaining hair.

Rabbits were placed on their left sides on a thin plastic membrane cut from a polyethylene bag stretched over an aluminum carrier frame as described previously (Fig. 1). ${ }^{11}$ The rabbits were then partially submerged in a tank of degassed water containing the therapeutic transducer and positioned so that the lower pole of the left kidney was colocalized with the focal zone of the transducer. Vital signs (heart rate, $\mathrm{SpO}_{2}$, and ventilatory rate) were monitored with a veterinary monitoring system (Heska Corp., Fort Collins, CO).

Ultrasound treatment consisted of 60,000 15-cycle (20- $\mu \mathrm{sec})$ bursts delivered to the lower pole of the left kidney at a rate of $1 \mathrm{kHz}$ from a 750-kHz transducer. Electronic steering was used to move the focal position depth rapidly by $5 \mathrm{~mm}$ for alternate bursts so that a total of 30,000 bursts was delivered to each location. The total treatment time was 60 seconds, and the cumulative ultrasound dose was equivalent to 1.2 seconds of continuous energy.

The rabbits were allowed to breathe freely. The motion of the kidney during treatment as observed by real-time ultrasound imaging was primarily in the craniocaudal direction and usually much less than $5 \mathrm{~mm}$. However, occasional excursions of as much as $10 \mathrm{~mm}$ did occur. Treatment was not gated to breathing.

In most cases, a bubble cloud was visible immediately by ultrasonography in the therapeutic focal region when ultrasound delivery started. If nothing was observed within the first $10 \mathrm{sec}-$ onds, the treatment was stopped and restarted at higher power: $24 \mathrm{MPa} \mathrm{P}_{\mathrm{R}}, 51.5 \mathrm{~kW} / \mathrm{cm}^{2}$.

After ultrasound treatment, the rabbits were moved to a heated table while they recovered. Total preparation and treatment time was 30 to 45 minutes, with anesthesia persisting about 1 hour. The day 0 rabbits were not allowed to recover and were instead euthanized, the kidneys being removed within 15 minutes of ultrasound treatment. The remaining rabbits were euthanized at the appropriate time points $(1,2,7,21$, or 60 days). One rabbit intended as part of the day 2 set died during recovery and was included with the day 0 rabbits. Thus, there were four rabbits in the day 2 group, whereas every other group consisted of five rabbits.

The treated kidneys were placed in Formalin. Once fixed, the kidneys were bivalved, and each half was processed. Sections cut at regular intervals were mounted and stained with either hematoxylin and eosin (H\&E) or Masson's trichrome (MT). The dimensions of the completely disrupted acellular material were measured for each kidney from the stained section showing the greatest cross-sectional area of injury.

\section{RESULTS}

Clear ultrasound access to the lower pole of the left kidney without rib obstruction was possible for all 29 animals. In 26

FIG. 2. Histologic effects of histotripsy. (Plate 1) Selected findings on days 0 through 2. All slides stained with H\&E. Area of disruption partially filled with hemorrhage is present on all days $(\mathbf{A}, \mathbf{D}, \mathbf{I})$. Disruption boundary is sharp, with some bordering karyorrhetic cells $(\mathbf{C})$ surrounded by small satellite areas of disruption and hemorrhage (B) up to a few millimeters. After 1 or 2 days, regions of necrosis, congestion, and tubular casts are apparent $(\mathbf{H}, \mathbf{K}, \mathbf{J})$. Neutrophils are seen infiltrating disruption border $(\mathbf{F})$. Some tubules and glomeruli are dilated $(\mathbf{G})$ compared with control region at opposite end of kidney (E). (Plate 2) Selected findings on days 7 through 60. The MT stain was used for $\mathbf{B}, \mathbf{D}, \mathbf{N}$, and $\mathbf{O}$ and H\&E for others. After 7 days, rim of fibroblasts and collagen deposition (pale blue) is present around disruption zone (A, B, D). Disruption zone is partially cystic. Areas of calcification $(\mathbf{C})$ and inflammatory remnants $(\mathbf{E})$ are present. After 21 days, disruption has been partially replaced with scar tissue $(\mathbf{J})$, and some macrophages are present $(\mathbf{H})$. Left contour defect caused by shrinkage $(\mathbf{F})$. Some dilation, calcification, and fibrosis are present in areas near disruption (G) having sharp boundary with normal tissue (I). After 60 days, contour defects $(\mathbf{K})$, dilation, and fibrosis persist $(\mathbf{L}, \mathbf{N})$. Parts $\mathbf{L}$ and $\mathbf{M}$ are from an H\&E-stained slide in and outside dilation area; $\mathbf{N}$ and $\mathbf{O}$ are from MT-stained slide of tissue from approximately the same locations. Significant collagen is indicated by blue stain $(\mathbf{N})$. 


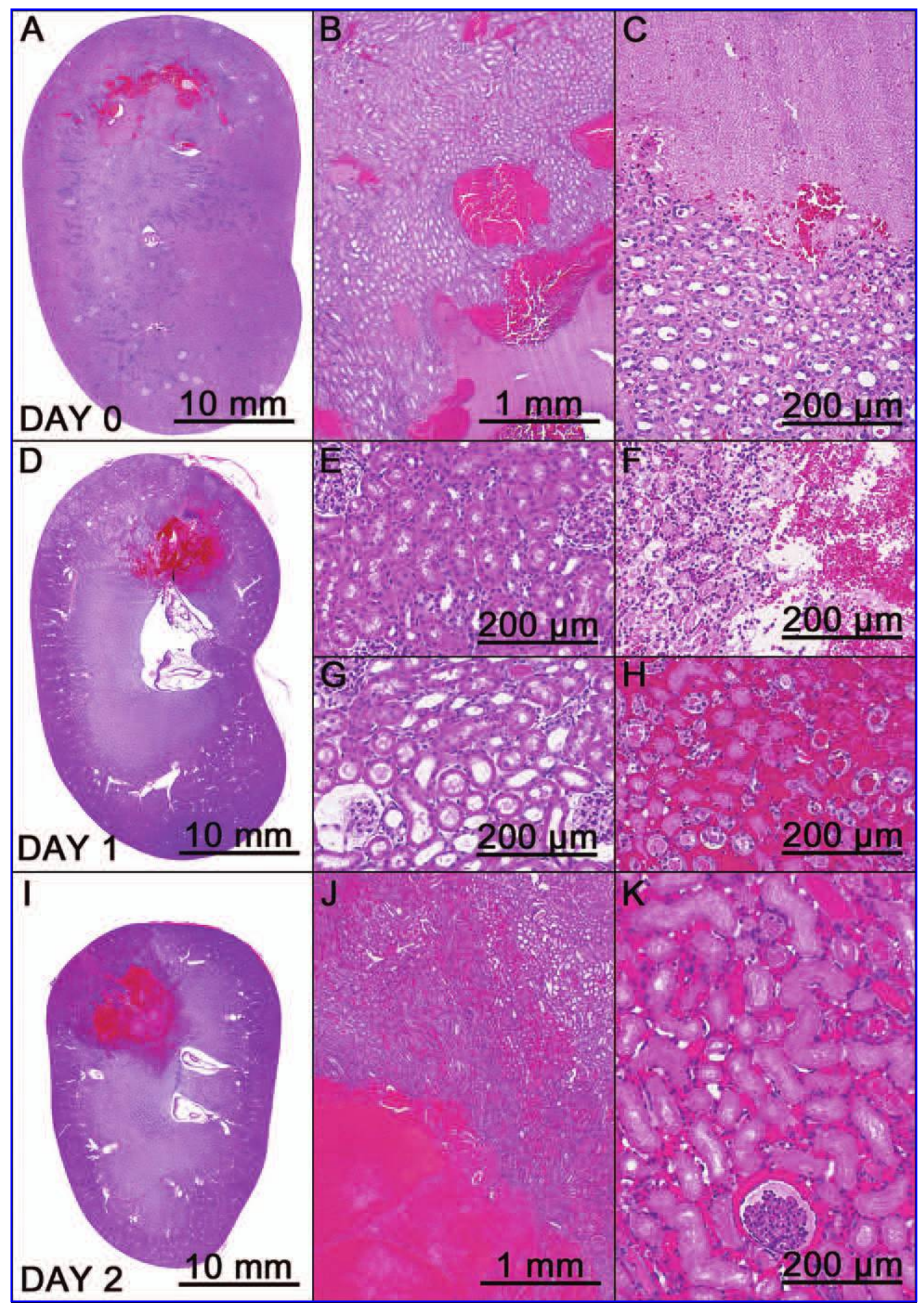




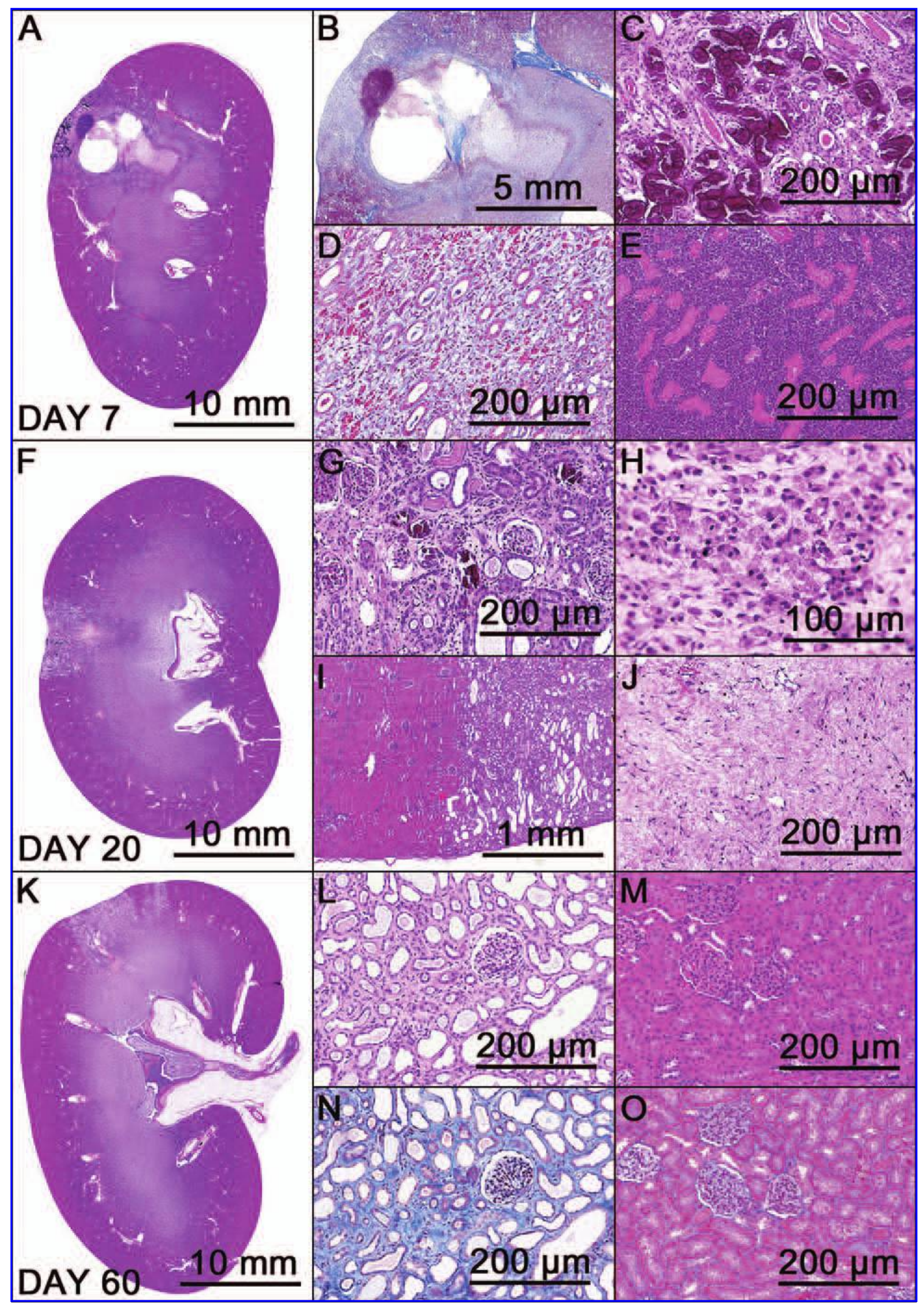

FIG. 2. (Continued). Plate 2 
cases, a region of highly transient hyperechogenicity (presumably a bubble cloud) was observed at the focus immediately on the start of treatment. For the other three cases, nothing was observed during the first 10 seconds. Following an increase in ultrasound amplitude, a cloud was observed.

Heart-rate increases were noted in some animals during treatment; however, the rates remained in the normal range of 150 to 250 beats/min at all times. All rabbits completed treatment and were in stable condition before being moved to the recovery location. One rabbit stopped breathing during the recovery period and could not be revived. The cause of death was not known, and exploration revealed no gross hemorrhage in the treatment region. The other 28 rabbits appeared healthy with no change in food intake. Hematuria occurred for the first 24 hours after treatment. Skin petechiae overlying the treatment location appeared in several animals but resolved after 1 day. Minor skin irritation (dryness, flaking) was present in some rabbits but frequently was not located at the site of ultrasound exposure and so was believed to have been caused by shaving. A significantly larger region of the abdomen had been shaved than was required for direct ultrasound propagation.

In kidneys harvested acutely (day 0), a contiguous area of finely disrupted tissue was observed containing no recognizable cells or cellular components. Red blood cells were observed in the lesion, although gross hemorrhage was not present. Along the boundary of architectural disruption, a border, several tubules in width, contained cells that were not visibly disrupted but contained pyknotic nuclei. Smaller satellite areas of disruption and hemorrhage were observed around the central disruption as much as $2 \mathrm{~mm}$ from the main site (Fig. 2 left). In kidneys harvested on day 1 or 2 , a rim of neutrophils was seen surrounding the disrupted area, and regions of tubular congestion and red blood cell casts were apparent (Fig. 2 right). By 7 days after treatment, fibrin, macrophages, and neutrophil remnants were seen in the disrupted area. Calcification also was noted, along with significant areas of dilated tubules and glomeruli with a regenerative appearance for tubular epithelium (Fig. 2 right). By day 21, the disrupted area had been mostly replaced by scar tissue and appeared contracted, leaving a defect in the kidney surface. Kidneys harvested on day 60 exhibited a small fibrous scar with an associated wedge-shaped region of tubular dilation and fibrosis extending to the capsule (Fig. 2 right). A surface contour defect was present suggestive of focal parenchymal loss. The measured area of complete cellular and architectural disruption steadily decreased in size over time, as shown in Figure 3.

\section{DISCUSSION}

For extirpative surgical procedures, confirmation of complete tumor resection is achieved by pathologic assessment of the specimen and resection margins. Subsequent surveillance for recurrent disease often involves cross-sectional imaging. However, for ablative modalities, extensive pathologic assessment is not possible, as the treated tissue is not removed from the body. A greater emphasis is therefore placed on cross-sectional imaging to assess the adequacy of ablation and recurrence of tumor. Unfortunately, interpretation of these studies often is confounded by the persistence of nonviable ablated tis-

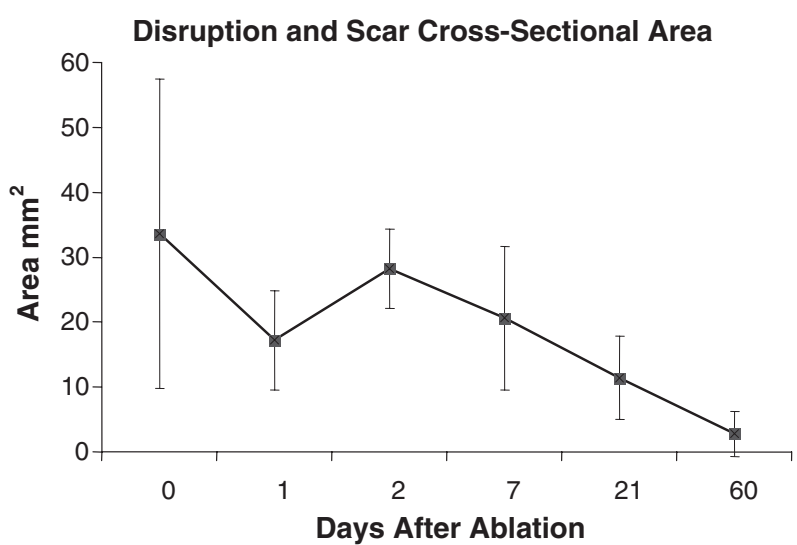

FIG. 3. Mean cross-sectional areas of disruption and scar tissue with time. Error bars represent standard deviations in measurements. Affected area shrinks over time commensurate with reabsorption and loss in volume. Note that time scale is irregular.

sue that itself can appear to be a mass. In a comparative study at the Cleveland Clinic, ${ }^{12}$ renal masses treated with RFA did not decrease in size even 2 years after ablation, and an average of $68 \%$ of the volume of cryolesions was still present 2 years after treatment.

Histologic analysis of RFA lesions immediately after ablation demonstrates hypereosinophilia, pyknotic nuclei, interstitial hemorrhage, and loss of cell-border integrity. ${ }^{13,14}$ However, the renal parenchymal architecture is preserved. ${ }^{15}$ Crowley and colleagues ${ }^{16}$ reported that at 3 and 13 weeks after ablation in an animal model, a central region of complete necrosis was surrounded by concentric bands of inflammatory infiltrate, hemorrhage, and fibrosis. High-intensity focused ultrasound also induces coagulative necrosis within a small cigar-shaped focal zone. However, ablating a clinically significant volume of tissue requires mechanical translation of the transducer to pack together a number of focal zones. Experiments with ex-vivoperfused porcine kidneys have been successful only with meticulous adjustment of exposure parameters. ${ }^{17}$ Frequently, this strategy results in patchy ablation, with cellular viability persisting between focal zones. ${ }^{17}$ With cryoablation in an animal model, immediate tubular necrosis, poorly defined cell borders, ${ }^{18}$ and interstitial hemorrhage ${ }^{19}$ evolve into a similar chronic appearance. One week after treatment, four distinct concentric zones can be identified: a central zone of complete necrosis, a zone of inflammatory infiltrate, a zone of hemorrhage, and an outer zone of fibrosis and regeneration. ${ }^{19}$

Histotripsy mechanically fractionates tissue and cellular structures, producing a fine acellular liquid or paste that is reabsorbed rapidly, as demonstrated in this study. Therefore, identification of residual tumor after histotripsy treatment may be simplified, as all sufficiently treated tissue is likely to be reabsorbed within 60 days.

Although ventilatory motion was not controlled for in this study, the disruption boundary is exceptionally sharp (on the order of one tubule), suggesting a mechanism of action less than a few tens of micrometers, which is consistent with the size of the resonant bubbles at $750 \mathrm{kHz} .{ }^{20}$ However, the overall treat- 
ment margin, as determined by the furthest extent of acutely visible effects, seems to be a few millimeters. A larger treatment volume created by scanning the focus should maintain the same margin around the edges of the disruption such that the margin would become a smaller fraction of the affected tissue. Additionally, as histotripsy fractionates tissue nonthermally, heat-sink effects from blood perfusion are not expected to alter the planned treatment zone.

Mechanical tissue bioeffects in the kidney also have been studied in the setting of shockwave lithotripsy (SWL). ${ }^{21-26} \mathrm{Al}-$ though in these studies, energy was delivered with lower intensities and many fewer pulses, and complete tissue fractionation was not observed, some histologic similarities are seen. After treatment with an electrohydraulic lithotripter in an animal model, scattered hemorrhage, tubular dilation, and tubular destruction were observed. Four weeks after treatment, the kidneys had regions of fibrosis, tubular dilation, and focal calcification. ${ }^{24}$ Piezoelectric ultrasound generators with much smaller focal zones than electrohydraulic generators were used by Prat and collaborators ${ }^{27}$ and Tavakkoli et $\mathrm{al}^{28}$ to create lesions in rabbit livers. These devices generated single-cycle shockwave pulses at low repetition rates. Hepatocyte disruption and hemorrhage were seen acutely, whereas fibrosis and typical inflammatory responses were reported after 21 days. However, complete cellular and architectural fractionation with generation of a liquefied material throughout the targeted zone was not seen.

It is important to understand that the potential of histotripsy to promote metastasis during treatment of neoplastic disease is unknown. Although SWL is distinct in terms of energy delivery and tissue bioeffects, it has been studied in association with metastasis in tumor models. Most of the studies evaluating SWL for tumor treatment have not shown higher rates of metastasis. However, several studies have suggested a stimulatory effect. ${ }^{25,26}$ One recent study assessed the rate of lung metastasis from SWL treatment of melanoma that had been implanted in the thigh of a mouse and injected with cavitation nuclei (contrast-agent microbubbles). ${ }^{25}$ Although the baseline rate of metastasis in the control animals was high (53\%) and histologic assessment of the degree of tumor treatment was not performed, a higher rate of metastasis was reported with SWL in the presence of microbubbles. Although the behavior of highly metastatic melanoma implanted in the thigh is not similar to that of renal-cell carcinoma, these results raise concerns about cavitational induction of tumor metastasis. Studies specifically addressing histotripsy treatment of tumor models are planned. Furthermore, it is important to study histotripsy in a larger animal model where a more substantial volume of tissue could be treated without destroying the entire kidney and cross-sectional imaging could be correlated as well. Despite these limitations, the reabsorption noted is encouraging.

\section{CONCLUSIONS}

Histotripsy is a focused ultrasound ablative modality that utilizes nonthermal mechanical (cavitational) processes to fractionate tissue architecture and cellular structures. Transcutaneous tissue fractionation was successful in a rabbit model, with sharp boundaries being apparent between the zone of complete fractionation and normal-appearing structures. The acellular material resulting from histotripsy treatment is readily reabsorbed by the body within 60 days in this rabbit model. This may prove to be a significant advantage for imaging assessment of residual tumor after ablation of renal malignancies.

\section{REFERENCES}

1. Jemal A, Siegel R, Ward E, et al. Cancer statistics, 2007. CA Cancer J Clin 2007;57:43-66.

2. Kennedy JE, ter Haar GR, Cranston D. High intensity focused ultrasound: Surgery of the future? Br J Radiol 2003;76:590-599.

3. Blana A, Walter B, Rogenhofer S, Wieland WF. High-intensity focused ultrasound for the treatment of localized prostate cancer: 5Year experience. Urology 2004;63:297-300.

4. McDannold N, Hynynen K. Quality assurance and system stability of a clinical MRI-guided focused ultrasound system: Four-year experience. Med Phys 2006;33:4307-4313.

5. Wu F, Wang Z, Chen W, Bai J, Zhu H, Qiao T. Preliminary experience using high intensity focused ultrasound for the treatment of patients with advanced stage renal malignancy. J Urol 2003; 170:2237-2240.

6. Kohrmann KU, Michel MS, Gaa J, Marlinghaus E, Alken P. High intensity focused ultrasound as noninvasive therapy for multilocal renal cell carcinoma: Case study and review of the literature. J Urol 2002;167:2397-2403.

7. Visioli AG, Rivens IH, ter Haar GR, et al. Preliminary results of a phase I dose escalation clinical trial using focused ultrasound in the treatment of localized tumours. Eur J Ultrasound 1999;9:11-18.

8. Tran BC, Seo J, Hall TL, Fowlkes JB, Cain CA. Microbubble-enhanced cavitation for noninvasive ultrasound surgery. IEEE Trans Ultrason Ferroelectr Freq Control 2003;50:1296-1304.

9. Xu Z, Fowlkes JB, Rothman ED, Levin AM, Cain CA. Controlled ultrasound tissue erosion: The role of dynamic interaction between insonation and microbubble activity. J Acoust Soc Am 2005;117: 424-435.

10. Parsons JE, Cain CA, Fowlkes JB. Characterizing pulsed ultrasound therapy for production of cavitationally-induced lesions. Proc 4th Int Symp Ther Ultrasound, 2004

11. Roberts WW, Hall TL, Ives K, Wolf JS Jr, Fowlkes JB, Cain C. Pulsed cavitational ultrasound: A noninvasive technology for controlled tissue ablation (histotripsy) in the rabbit kidney. $\underline{\mathrm{J} \text { Urol }}$ 2006;175:734-738.

12. Hegarty NJ, Gill IS, Desai MM, Remer EM, O’Malley CM, Kaouk $\mathrm{JH}$. Probe-ablative nephron-sparing surgery: cryoablation versus radiofrequency ablation. Urology 2006;68(supp 1A):7-13.

13. Zlotta AR, Wildshutz T, Raviv G, et al. Radiofrequency interstitial tumor ablation (RITA) is a possible new modality for treatment of renal cancer: Ex-vivo and in-vivo experience. J Endourol 1997; 11:251-258.

14. Hsu THS, Fidler ME, Gill IS. Radiofrequency ablation of the kidney: Acute and chronic histology in porcine model. Urology 2000;56:872-875.

15. Corwin TS, Lindberg G, Traxer O, et al. Laparoscopic radiofrequency thermal ablation of renal tissue with and without hilar occlusion. J Urol 2001;166:281-284.

16. Crowley JD, Shelton J, Iverson AJ, Burton MP, Dalrymple NC, Bishoff JT. Laparoscopic and computed tomography-guided percutaneous radiofrequency ablation of renal tissue: Acute and chronic effects in an animal model. Urology 2001;57:976-980.

17. Hacker A, Peters K, Knoll T, et al. Ablation of clinically relevant kidney tissue volumes by high-intensity focused ultrasound: Preliminary results of standardized ex-vivo investigations. 2006;20:930-938. 
18. Nakade SY, Lee FT, Warner T, Chosy SG, Moon TD. Laparoscopic cryosurgery of the kidney in the porcine model: An acute histological study. Urology 1998;51(suppl 5A):161-166.

19. Bishoff JT, Chen RB, Lee BR, et al. Laparoscopic renal cryoablation: Acute and long-term clinical, radiographic, and pathologic effects in an animal model and application in a clinical trial. J Endourol 1999;13:233-239.

20. Leighton TG: The Acoustic Bubble. London: Academic Press, 1994, pp 295-297.

21. Delius M, Enders G, Xuan Z, Liebich HG, Brendel W. Biological effects of shock waves: Kidney damage by shock waves in dogs: Dose dependence. Ultrasound Med Biol 1988;14:117-122.

22. Delius M, Jordan M, Eizenhoefer H, Marlinghaus E, Heine G, Liebich HG, Brendel W. Biological effects of shock waves: Kidney haemorrhage by shock waves in dogs: Administration rate dependence. Ultrasound Med Biol 1988;14:689-694.

23. Willis LR, Evan AP, Connors BA, et al. Shockwave lithotripsy: Dose-related effects on renal structure, hemodynamics, and tubular function. J Endourol 2005;19:90-101.

24. Newman R, Hackett R, Senior D, et al. Pathologic effects of ESWL on canine renal tissue. Urology 1987;29:194-200.

25. Oosterhoff GON, Cornel EB, Smits GAHJ, Debruyne FMJ, Schalken JA. The influence of high-energy shock waves on the development of metastases. Ultrasound Med Biol 1996;22:339-344.

26. Miller DL, Dou C, Song J. Lithotripter shockwave-induced enhancement of mouse melanoma lung metastasis: Dependence on cavitation nucleation. J Endourol 2004;18:925-929.
27. Prat F, Chapelon JY, Abou F, Sibille A, Theillière Y, Ponchon T, Cathignol D. Focused liver ablation by cavitation in the rabbit: A potential new method of extracorporeal treatment. Gut 1994; 35:395-400.

28. Tavakkoli J, Birer A, Arefiev A, Prat F, Chapelon JY, Cathignol D. A piezocomposite shock wave generator with electronic focusing capability: Application for producing cavitation-induced lesions in rabbit liver. Ultrasound Med Biol 1997;23:107-115.

Address reprint requests to: William W. Roberts, M.D.

Dept. of Urology University of Michigan TC 3879

1500 East Medical Center Drive Ann Arbor, MI 48109-0330

E-mail: willrobe@umich.edu

\section{ABBREVIATIONS USED}

$\mathrm{H} \& \mathrm{E}=$ hematoxylin and eosin; MT = Masson's trichrome; $\mathrm{RFA}=$ radiofrequency ablation; $\mathrm{SWL}=$ extracorporeal shockwave lithotripsy. 

This article has been cited by:

1.2008. Abstracts of the Engineering and Urology Society, May 17, 2008, Orlando, FloridaAbstracts of the Engineering and Urology Society, May 17, 2008, Orlando, Florida. Journal of Endourology 22:11, 2583-2640. [Citation] [PDF] [PDF Plus] 